\title{
Reliability of computer-assisted surgery as an intraoperative ruler in navigated high tibial osteotomy
}

\author{
Florian Gebhard • Christian Krettek • Tobias Hüfner • Paul A. Grützner • Ulrich Stöckle • \\ Andreas B. Imhoff · Stephan Lorenz · Jan Ljungqvist • Peter Keppler • The AO CSEG
}

Received: 18 November 2009/Published online: 6 July 2010

(c) The Author(s) 2010. This article is published with open access at Springerlink.com

\begin{abstract}
Background Computer-assisted surgery (CAS) can act as an intraoperative ruler in high tibial osteotomy (HTO) to visualize continuously the leg during surgery.

Questions The aim of the study is to evaluate the accuracy of CAS with respect to preoperative planning and postoperative deviation from the planned leg axis in HTO. In addition,
\end{abstract}

Members of the AO Computer-Assisted Surgery Expert Group:

C. Krettek, T. Hüfner, P. Messmer, U. Stöckle, D. Kahler,

P. A. Grützner.

Supported by AOCID.

F. Gebhard $(\bowtie) \cdot$ P. Keppler

Department for Orthopaedic Trauma, Ulm University,

Steínhövelstrasse 9, 89075 Ulm, Germany

e-mail: florian.gebhard@uniklinik-ulm.de

C. Krettek · T. Hüfner

Trauma Department, Hannover Medical School,

Carl-Neuberg-Strasse 1, 30625 Hannover, Germany

P. A. Grützner

Clinic for Orthopaedic and Trauma Surgery,

Klinikum Stuttgart-Katharinenhospital, Kriegsbergstrasse 60,

70174 Stuttgart, Germany

U. Stöckle

Department for Trauma Surgery, Klinikum rechts der Isar,

Technical University Munich, Ismaningerstrasse 22,

81675 Munich, Germany

A. B. Imhoff · S. Lorenz

Department of Orthopaedic Sport Surgery,

Klinikum rechts der Isar, Technical University Munich,

Connollystrasse 32, 80809 Munich, Germany

J. Ljungqvist

AO Clinical Investigation and Documentation,

AO Foundation, Clavadelerstrasse 8, 7270 Davos, Switzerland the influence of surgeon experience as well as operation time and perioperative complications are analyzed.

Methods A prospective multicenter study case series with follow-up at 6 weeks was performed in six centers. Medial open-wedge HTO with Tomofix ${ }^{\circledR}$ was done using computer assisted navigation technique with the Brainlab VV Osteotomy 1.0 module.

Results Fifty-one patients with medial gonarthritis were treated with navigated HTO. The follow-up rate was $98 \%$. The majority of HTO-CAS patients fell within the tolerated limit of $\pm 3^{\circ}$ for leg axis deviation, however, seven patients were reported with deviations outside of this range: three patients had deviations of $>3^{\circ}-4.5^{\circ}$ and four patients $>4.5^{\circ}$, respectively. Eight intraoperative complications were documented, partially resulting from technical problems associated with the navigation system. During the 6-week follow-up period, three postoperative complications were experienced, all not associated with navigation technology. Conclusions In about $85 \%$ of cases, a perfect result in terms of deviation of the planned mechanical leg axis could be achieved. Computer assistance in HTO proved to be a helpful tool regarding intraoperative control of leg axis.

Level of evidence Level I, High quality prospective study (all patients were enrolled at the same preoperative planning point with $\geq 80 \%$ follow-up of enrolled patients).

Keywords Knee - Tibia - Osteotomy - Surgery, computer-assisted $\cdot$ Bone and bones $\cdot$ Navigation . Planning

\section{Introduction}

Today the most common application in the field of computer-assisted surgery is navigated total knee arthroplasty. 
As several prospective randomized studies could show, the standard deviation of the mechanical leg axis is reduced significantly $[4,14,16]$. The mechanical leg axis is one important factor influencing the mechanical load distribution in the knee joint. According to the biomechanical studies of Hsu et al. [6], in a $1^{\circ}$ varus deformity $75 \%$ of the knee joint load passes through the medial tibial plateau but this load increases to over $90 \%$ in a $6^{\circ}$ varus deformity.

An effective method to decrease the load of the medial tibial plateau is a valgisation of the leg. High tibial osteotomy (HTO), first reported by Jackson in 1961 [8] and later popularized by Coventry [2] in the United States, is a common procedure in the management of medial compartment osteoarthritis (OA) of the knee and is often reported as an effective method of management $[5,7,9]$.

In principle, two different techniques are available: (1) the closed-wedge technique, and (2) the open-wedge technique. The medial open-wedge HTO is considered to be superior to the closed-wedge procedure in terms of leaving the fibular bone unaltered. It also does not lead to a loss of bone and is assumed to encounter less iatrogenic soft tissue damage [5, 10, 15].

The TomoFix ${ }^{\circledR}$ implant was designed to overcome previous plate limitations in performing medial wedge HTO. According to the designers of this plate and screw system, the difference from conventional systems is that the screws can be locked in the plate hole, which provides angle stability and no primary or secondary tilting of the fragment [17]. These advantages are believed to prevent primary and secondary loss of correction and ultimately, improved functional outcomes [11, 17].

Ideal correction of the leg axis is difficult to achieve and postoperative malalignment is observed following HTO [3, 12]. A permanent surgical challenge is to achieve the planned leg axis intraoperatively. Computer-assisted navigation systems may improve precision and accuracy of the leg-axis correction, while offering simulation tools and being capable of predicting the postoperative alignment.

The hypothesis of this study is that CAS guidance in HTO is a reliable tool with respect to preoperative planning and postoperative deviation from the planned.

\section{Patients and methods}

Study design

After approval of local ERBs, a prospective case series was conducted including patients with medial gonarthritis or genu varum congenitum undergoing medial open-wedge HTO using computer-assisted navigation at six European trauma centers.
A majority of 51 patients were recruited in Klinikum rechts der Isar, Technical University Munich $(n=17)$, followed by Ulm University ( $n=14)$, BGU-Ludwigshafen $(n=9)$, Hannover Medical School $(n=8)$, Charité Berlin-Campus Virchow Klinikum $(n=2)$ and Klinikum Stuttgart-Katharinenhospital $(n=1)$.

\section{Relevant patient demographics}

Patients were included if they were aged 18 years or older and provided written informed consent, as well as commitment to attend the planned follow-up prior to participation. In addition, those candidates with an intact lateral joint compartment, as well as physiological age/appropriate range of motion in the hip, knee or ankle of the affected leg were included for medial open-wedge HTO surgery. Exclusion criteria included patients with a body mass index $\geq 35$, a history of drug or alcohol abuse, bone healing disorders, the presence or history of active malignancy or systemic disease, physical or mental incapacity, legal incompetence, systemic or severe local inflammation or infections, and lastly any patient receiving radio- or chemotherapy before, during or within a period of 1 year.

This analysis including 46 patients had $91 \%$ power to identify a relevant deviation from $0^{\circ}$ of $1^{\circ}$ at the 0.05 significance level, assuming a standard deviation of angle of $2^{\circ}$.

\section{Description of surgery}

The surgical technique is described in detail in the Synthes Tomofix ${ }^{\text {TM }}$ Osteotomy System technique guide. Navigation was carried out using the Brainlab VectorVision Osteotomy module Version 1.0. (FDA 042513).

Minimally invasive reference array units are mounted ideally in the medial cortex of the tibia and ventral medial or ventral lateral side of the femur. Registration automatically starts with the definition of the center of the femoral head, which is found by pivoting the leg in the hip joint. When a precision of less than $2 \mathrm{~mm}$ is achieved, the software automatically proceeds to the next step. Anatomical landmarks to calculate the center of the knee and ankle are registered percutaneously with a pointer. The first landmark is the medial malleolus followed by the lateral malleolus. The medial tibial plateau border is registered, as well as the lateral tibial plateau border. The anteroposterior (AP) direction is then defined. The last points to be digitalized are the medial and lateral epicondyle. The surgeon now has all the information on the mechanical leg axis, the degree of flexion, and the relative rotation of the tibia against the femur. On the screen of the navigation system the leg is shown with the alignment parameters. Planning is 


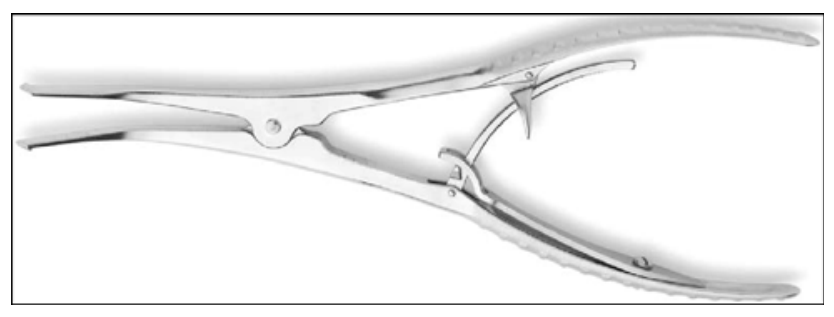

Fig. 1 Spreader that enables the surgeon to continuously open the osteotomy until the planned value is achieved

Fig. 2 Tomofix ${ }^{\mathrm{TM}}$ plate that allows fixing the osteotomy in the exact position as necessary to achieve the planned leg axis. Head locking screws prevent loss of position after removing the spreader

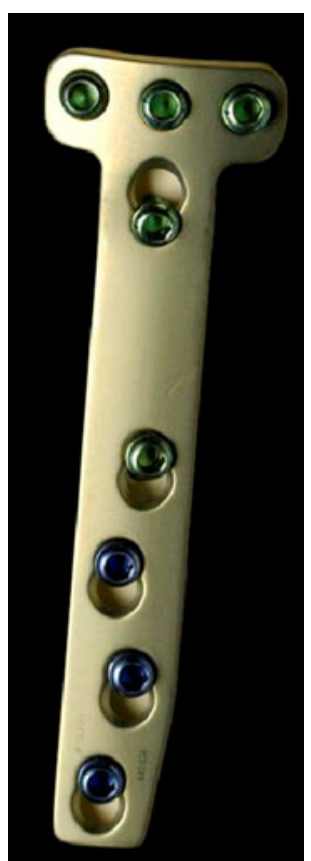

finalized by defining the amount of leg axis correction needed based on the preoperative plan.

After planning, K-wires are positioned by navigating the drill guide to the right height and angulation. Two K-wires guide the saw in the predetermined direction. The planned depth of the cut is shown on the navigation screen and can be checked when cutting. A spreading device (spreader, Synthes (C), Fig. 1) is used from the medial side to continuously correct the leg axis. The navigation screen displays information in real time. The fixation is also done under navigation control, and so any possible loss of correction can be detected and easily corrected. After correct leg alignment, the osteotomy is fixed with the Synthes Tomofix $^{\text {TM }}$ plate (Fig. 2).

\section{Postoperative treatment}

Postoperative treatment in all patients included radiographic control as well as partial weight bearing for 6 weeks with unrestricted extension/flexion of the knee joint.
Follow-up routine

During hospitalization, patient demographics (i.e., gender, age, height, weight, smoking) and baseline characteristics (i.e., date of examination, affected side and type of deformity, general pre-existing disorders, range of motion for the hip, knee and ankle of the affected and contralateral leg, ligament stability of both knees, surgeon characteristics, operation time and details, implant characteristics) were recorded. Intraoperative complications such as iatrogenic fractures, bleeding, technical and surgical problems were documented. Patients were $\mathrm{X}$-rayed in AP (long-standing X-ray) and lateral projection (knee joint) at their initial attendance, as well as postoperatively.

Patients were actively followed-up after 6 weeks. At this follow-up, a long standing X-ray and an X-ray of the knee joint in two planes were performed to survey leg axis, fracture healing and the possible occurrence of complications. The prerequisites for long standing X-rays were full weight bearing and no active extension deficit more than $5^{\circ}$ of the affected knee joint. Postoperative complications (adverse events) were documented throughout the postoperative period up to 6 weeks. Anticipated complications included unplanned reoperation, implant problems such as secondary implant dislocation, implant loosening, breakage and failure, as well as healing problems and other local or general complications such as wound infection or hematoma, systemic infections, thromboembolic complications, or fat embolism. Reported complications and related patient X-rays were reviewed by an independent surgeon to define if complications were mild, moderate or serious, and occurred due to the implant, navigation or as a result of the patients' general condition.

Patients were interviewed concerning their walking ability (PMS), their pain at rest and during motion (WOMAC pain subscale) [1], as well as their satisfaction with current function (VAS) before surgery and at the 6-week follow-up. Range of motion of the hip, knee and ankle, ligament stability of the knee, as well as current mobilization status were determined at the follow-up examination.

\section{Outcome measures}

The measurements of the planned and actual postoperative leg axis deviations for patients undergoing HTO were made using the following parameters according to the classification of Paley et al. [13] that are listed below:

- mFTA (mechanical femorotibial angle, mechanical leg axis) was used as the main parameter to measure the primary outcome. 
- mMPTA (mechanical medial proximal tibial angle) was used to evaluate whether the indication for HTO was correct.

- mLDFA (mechanical lateral distal femoral angle) was used to control for the measurement reliability in the $\mathrm{X}$-ray projection.

For the planned postoperative leg axis measurement, the individual centers were required to assess this parameter from preoperative X-rays.

Prior to surgery, a fax sheet with the pre- and planned postoperative angles was sent to AO Clinical Investigation and Documentation Center (AOCID), Davos/Switzerland for final evaluation. At 6 weeks follow-up examination, the actual ("effective") leg axis measurement was assessed by an independent surgeon from all postoperative long standing X-rays using the MediCAD software.

The final outcome measurement of precision of the achieved leg axis was equivalent to the deviation of the effective leg axis from the planned postoperative leg axis (i.e., actual "effective" postoperative leg axis-planned postoperative leg axis).

Statistical analysis

Study monitoring, database management (including data entry), plausibility checks and query generation, and statistics were performed by AOCID. Data were entered into an OpVerdi Database (Dr. Oestreich and Partner, GmbH, Cologne, Germany) and transferred via text files into the software Intercooled Stata Version 10 (StataCorp LP, College Station, Texas, USA) for statistical analysis.

All baseline and follow-up parameters were described using standard descriptive statistics. While continuous variables were described using means, standard deviations and ranges, categorical variables were tabulated with absolute and relative frequencies.

The primary outcome of leg axis deviation was analyzed using the one-sample $t$ test to test the null hypothesis that the mean deviation was equal to zero. The percentage of patients with a deviation greater than $3^{\circ}$ was calculated.

All statistical analyses and graphs were conducted with the software Intercooled Stata Version 10.

\section{Results}

Fifty-nine patients were included in the study. Due to major study protocol violations, 8 patients needed to be excluded for various reasons, which led to a final number of 51 cases receiving navigated HTO recruited in six centers between January 2006 and October 2007. At the 6-week follow-up examination, 50 patients were examined and this corresponded to a follow-up rate of $98 \%$. Only one HTO-Navi patient did not attend the 6-week examination since their preoperative planning form was incomplete and as a result, the primary outcome could not be documented. HTO-Navi patients were seen for follow-up after a median of 6.6 weeks (range: 4.4-18.0) at the planned 6-week examination. Majority of these patients $(n=39)$ were examined within a threshold time range of $6 \pm 1$ weeks; only ten HTO-Navi patients attended the follow-up examination after 8.7-14.0 weeks and a single patient was seen at 18 weeks.

The demographic data of patients receiving navigated HTO is given in Table 1.

Navigated HTO surgery was mostly undertaken by consultants $(73 \%)$ followed by senior residents $(22 \%)$ and chief surgeons $(6 \%)$. Over $50 \%$ had performed 30 or more HTO procedures without navigation and between 10 and 29 HTO procedures with a navigation system.

The mean length of surgery time was 105 min (range: 60-200 min). Over 60\% (32/51) of the navigated HTO operations were completed within 1-2 $\mathrm{h}$.

In four patients, the quality of the long standing X-rays was not sufficient to measure the appropriate angles. Therefore, 46 patients were included in the following interpretation. The mean leg axis deviation was $1.5^{\circ}$ (standard error $=0.3$ ) and was found to be significantly different from zero ( $p<0.0001$; one-sample $t$ test). The minimum, median and maximum leg axis deviations were $-2.4^{\circ}, 1.8^{\circ}$ and $6.4^{\circ}$, respectively.

Table 1 HTO-Navi patient demographics and injury-related data at baseline

\begin{tabular}{lcccccc}
\hline Factors & $n$ & $\%$ & Mean & SD & Median & Range \\
\hline Age (years) & 51 & & 45.4 & 8.6 & 46.0 & $22.0-59.0$ \\
Gender & & & & & & \\
$\quad$ Male & 40 & 78 & & & & \\
Female & 11 & & & & & \\
Body mass index & 51 & & 27.5 & 3.0 & 27.7 & $21.2-34.5$ \\
Smoking & & & & & & \\
Yes & 11 & 22 & & & & \\
No & 40 & & & & & \\
Affected side & & & & & & \\
Right & 23 & 45 & & & & \\
Left & 28 & & & & \\
Prior surgery of affected leg & & & & \\
Yes & 42 & 82 & & & & \\
No & 9 & & & & \\
Type of deformity & & & & \\
Post-traumatic & 12 & 24 & & & \\
Congenital & 39 & & & \\
\hline
\end{tabular}

$S D$ standard deviation 


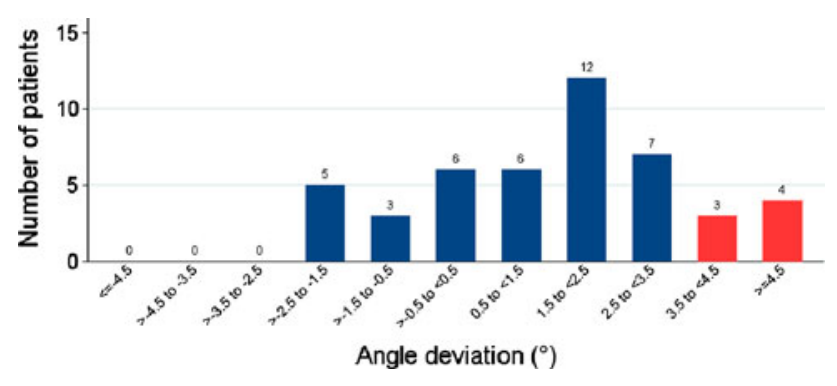

Fig. 3 Categorized distribution of the calculated leg axis deviation for HTO-Navi patients. All red bars indicate the patients for whom leg axis deviation was more than the tolerated threshold of $3^{\circ}$

The leg axis deviations categorized according to their degree of deviation (i.e., $>-0.5^{\circ}$ to $<0.5^{\circ}, 0.5^{\circ}-<1.5^{\circ}$, $1.5^{\circ}-<2.5^{\circ}$ etc.) are presented in Fig. 3 .

Deviations up to $3^{\circ}$ were tolerated and could be explained by the measurement deviations derived from the $\mathrm{X}$-rays and navigation software. Therefore, HTO-Navi patients with deviations of over $\pm 3^{\circ}$ were of special interest in this study.

Twenty-two patients (48\%) showed a leg axis deviation of up to $2^{\circ}\left(<2.5^{\circ}\right)$ and 39 patients $(85 \%)$ had deviations of up to $3^{\circ}\left(<3.5^{\circ}\right)$. Seven patients were categorized with leg axis deviations beyond the tolerance level of $3^{\circ}$ and included three patients with deviations $>3-4^{\circ}$ and four patients with deviations $>4^{\circ}$ (Fig. 3). Further analyses to observe any potential influence of medial ligament instability (i.e., patients with Grade II or III medial extension or flexion at baseline or the 6-week follow-up) on these cases of higher leg axis deviation revealed that there was no ligament instability for these particular patients.

Seven intraoperative complications were reported from a total of 59 patients at the beginning of the study (12\%). They were all derived from the navigation system and the majority occurred during one study center's learning phase. Loosening of the reference marker base was the most common intraoperative complication $(n=3)$, followed by system failure $(n=2)$, loss of orientation after changing the reference pins $(n=1)$ and the unavailability of the navigation instrument $(n=1)$. In all these patients, HTO surgery was continued in a conventional fashion.

Three postoperative complications were documented up to the 6-week follow-up examination, where two were defined as moderate soft tissue/wound complications and the third problem was a severe bone complication; all events were not directly related to the CAS procedure.

\section{Discussion}

In HTO, it is crucial to achieve a correct leg axis as defined in the preoperative planning. If the leg axis is under corrected, the transfer of weight from the medial to the lateral compartment is incomplete and the patient still experiences pain and the gonarthritis progresses. If it is overcorrected (too much valgus), the knee may become instable and the arthritis progresses faster on the lateral compartment.

Most authors recommend a postoperative valgus of $2^{\circ}-3^{\circ}$ based on long leg x-rays. Most important is the preoperative planning based on these films that give the size of the wedge, as during the operation the information of the long leg radiographs is not available. A conventional intraoperative control of the leg axis is the cable method, however, it is not always precise and quite an amount of radiation is necessary.

This is the first reported prospective multicenter case series focusing on the accuracy of navigated leg axis control in HTO. In contrast to other case series in navigated total knee replacement, in this study the preoperative definition of the aimed leg axis and submitting the latter to the study center was a must. Therefore, the results precisely describe the accuracy of system as the final leg axis deviates from a defined preoperative value given to the study center.

The study proves that computer assisted leg axis control is a very precise intraoperative guidance tool in osteotomies. The accuracy is extremely high compared to standard surgical procedure that relies on "surgeon's eye"- or fluoroscopy-controlled evaluation of the aimed leg axis. Even the intraoperative use of wedges is not that precise.

One limitation of our study is that the experience i.e., learning curve was different within the study centers. Dropout patients, i.e., deviation of more than $3^{\circ}$, are mainly caused by handling failures of the system such as loosening of reference arrays all of which occurred in one study center during its learning phase. But on the other hand, the results show that despite the learning curve the accuracy still is high.

If we would remove the four patients with a deviation of $>4^{\circ}$ that occurred within the learning phase of one study center, tolerable alignment would have been achieved in almost $93 \%$ of all cases.

Another limitation in all these studies focusing on leg axis is that the reported deviations from the planned leg axis are partially influenced by the fact that intraoperatively the final leg axis is evaluated on the resting leg whereas the postoperative X-ray control is done on the weight bearing leg which automatically means $1^{\circ}$ or $2^{\circ}$ of difference. In addition, the long standing $\mathrm{X}$-rays may give an error up to $2^{\circ}$ depending on full knee extension and inward/outward orientation of the leg. To reduce this additional error, all long standing X-rays have been analyzed for these technical aspects as well.

Regarding the correlation of radiographic and navigation measurement in limb alignment Stulberg and 
coworkers [18] recently reported a reasonable discrepancy as much as $8^{\circ}$ in reading out the results.

With respect to that finding our results prove that navigated HTO is a highly precise tool in defining intraoperative limb alignment.

The described limitations are the major reason to the fact that the accuracy of the achieved postoperative leg axis is not in all cases within the aimed range of $\pm 3^{\circ}$.

Most of the rated complications are surgery related and not due to computer navigation except for the cases of system down or software failure.

Our initial hypothesis could be proven as the study clearly shows that in this first prospective case series in about $85 \%$ (including learning curve), a perfect result in terms of deviation of the planned mechanical leg axis could be achieved using computer assistance as in intraoperative guiding tool. In the hands of trained CAS surgeons one might expect that the accuracy will be even higher (93\% excluding learning curve cases).

The study shows that in navigated HTO based on the described surgical technique a perfect intraoperative leg axis with respect to the preoperative plan can be achieved.

But nevertheless a navigation system is not a guarantee for a perfect result itself.

Open Access This article is distributed under the terms of the Creative Commons Attribution Noncommercial License which permits any noncommercial use, distribution, and reproduction in any medium, provided the original author(s) and source are credited.

\section{References}

1. Bellamy N, Buchanan WW, Goldsmith CH, Campbell J, Stitt LW (1988) Validation study of WOMAC: a health status instrument for measuring clinically important patient relevant outcomes to antirheumatic drug therapy in patients with osteoarthritis of the hip or knee. J Rheumatol 15:1833-1840

2. Coventry MB (1987) Proximal tibial varus osteotomy for osteoarthritis of the lateral compartment of the knee. J Bone Joint Surg Am 69:32-38

3. Dahl MT (2000) Preoperative planning in deformity correction and limb lengthening surgery. Instr Course Lect 49:503-509

4. Hart R, Janecek M, Chaker A, Bucek P (2003) Total knee arthroplasty implanted with and without kinematic navigation. Int Orthop 27:366-369
5. Hernigou P, Medevielle D, Debeyre J, Goutallier D (1987) Proximal tibial osteotomy for osteoarthritis with varus deformity. A ten to thirteen-year follow-up study. J Bone Joint Surg Am 69:332-354

6. Hsu RW, Himeno S, Coventry MB, Chao EY (1990) Normal axial alignment of the lower extremity and load-bearing distribution at the knee. Clin Orthop 255:215-227

7. Insall JN, Joseph DM, Msika C (1984) High tibial osteotomy for varus gonarthrosis. A long-term follow-up study. J Bone Joint Surg Am 66:1040-1048

8. Jackson JP, Waugh W (1961) Tibial osteotomy for osteoarthritis of the knee. J Bone Joint Surg Br 43-B:746-751

9. Jokio PJ, Lindholm TS, Vankka E (1985) Medical and lateral gonarthrosis treated with high tibial osteotomy. A prospective study. Arch Orthop Trauma Surg 104:135-144

10. Koshino T, Murase T, Saito T (2003) Medial opening-wedge high tibial osteotomy with use of porous hydroxyapatite to treat medial compartment osteoarthritis of the knee. J Bone Joint Surg Am 85A:78-85

11. Lobenhoffer P, Agneskirchner JD (2003) Improvements in surgical technique of valgus high tibial osteotomy. Knee Surg Sports Traumatol Arthrosc 11:132-138

12. Noyes FR, Barber-Westin SD, Hewett TE (2000) High tibial osteotomy and ligament reconstruction for varus angulated anterior cruciate ligament-deficient knees. Am J Sports Med 28:282-296

13. Paley D, Herzenberg JE, Tetsworth K, McKie J, Bhave A (1994) Deformity planning for frontal and sagittal plane corrective osteotomies. Orthop Clin North Am 25:425-465

14. Perlick L, Bathis H, Lerch K, Luring C, Tingart M, Grifka J (2004) Navigated implantation of total knee endoprostheses in secondary knee osteoarthritis of rheumatoid arthritis patients as compared to conventional technique. Z Rheumatol 63:140-146

15. Siguier M, Brumpt B, Siguier T, Piriou P, Judet T (2001) [Original valgus tibial osteotomy by internal opening and without loss of bone contact. Technique and incidence of consolidation speed: a preliminary series of 33 cases]. Rev Chir Orthop Reparatrice Appar Mot 87:183-188

16. Sparmann M, Wolke B, Czupalla H, Banzer D, Zink A (2003) Positioning of total knee arthroplasty with and without navigation support. A prospective, randomised study. J Bone Joint Surg Br 85:830-835

17. Staubli AE, De Simoni C, Babst R, Lobenhoffer P (2003) TomoFix: a new LCP-concept for open wedge osteotomy of the medial proximal tibia-early results in 92 cases. Injury 34(Suppl 2):B55B62

18. Yaffe MA, Koo SS, Stulberg SD (2008) Radiographic and navigation measurements of TKA limb alignment do not correlate. Clin Orthop Relat Res 466:2736-2744 